\title{
Can an aversive, extinction-resistant memory trigger impairments in walking adaptability? An experimental study using adult rats.
}

Filipe Mello Medeiros ${ }^{\mathrm{a}, \mathrm{b}, \mathrm{c}}$, Jociane de Carvalho Myskiw ${ }^{\mathrm{d}}$, Pedro Porto Alegre Baptista ${ }^{\mathrm{a}}$, Laura Tartari Neves ${ }^{\mathrm{a}, \mathrm{c}}$, Lucas Athaydes Martins ${ }^{\mathrm{a}, \mathrm{b}}$, Cristiane Regina Guerino Furini ${ }^{\mathrm{d}}$, Iván Izquierdo ${ }^{\mathrm{d}}$, Léder Leal Xavier ${ }^{\mathrm{a}, \mathrm{b}, \mathrm{c}}$, Kristen Hollands ${ }^{\mathrm{e}}$, Régis Gemerasca Mestriner ${ }^{\mathrm{a}, \mathrm{b}, \mathrm{c}}$.

${ }^{\text {a }}$ Cell and Tissue Laboratory, Biosciences College. Pontifícia Universidade Católica do Rio Grande do Sul (PUCRS), Brazil.

${ }^{\mathrm{b}}$ Neuroplasticity and Rehabilitation Research Group, Pontifícia Universidade Católica do Rio Grande do Sul (PUCRS), Brazil.

${ }^{\mathrm{c}}$ Graduate Program in Cellular and Molecular Biology, Pontifícia Universidade Católica do Rio Grande do Sul (PUCRS), Brazil.

${ }^{\text {d }}$ Memory Center, Brain Institute of Rio Grande do Sul, Pontifícia Universidade Católica do Rio Grande do Sul (PUCRS), Brazil.

${ }^{\mathrm{e}}$ Health Sciences College. University of Salford - Manchester, United Kingdom.

*Corresponding author:

Régis Gemerasca Mestriner, PhD.

Avenida Ipiranga, 6681

Prédio $12 / 8 .^{\circ}$ andar

Porto Alegre, RS, Brazil

CEP: 90619-900

e-mail: regis.mestriner@ pucrs.br

Phone: (55) (51) 33203646 


\begin{abstract}
Cognitive demands can influence the adaptation of walking, a crucial skill to maintain body stability and prevent falls. Whilst previous research has shown emotional load tunes goal-directed movements, little attention has been given to this finding. This study sought to assess the effects of suffering an extinction-resistant memory on skilled walking performance in adult rats, as an indicator of walking adaptability. Thus, 36 Wistar rats were divided in a two-part experiment. In the first part $(n=16)$, the aversive, extinction-resistance memory paradigm was established using a fear-conditioning chamber. In the second, rats $(n=20)$ were assessed in a neutral room using the ladder rung walking test before and tree days after inducing an extinction-resistance memory. In addition, the elevated plus-maze test was used to control the influence of the anxiety-like status on gait adaptability. Our results revealed the shock group exhibited worse walking adaptability (lower skilled walking score), when compared to the sham group. Moreover, the immobility time in the ladder rung walking test was similar to the controls, suggesting that gait adaptability performance was not a consequence of the fear generalization. No anxiety-like behavior was observed in the plus maze test. Finally, correlation coefficients also showed the skilled walking performance score was positively correlated with the number of gait cycles and trial time in the ladder rung walking test and the total crossings in the plus maze. Overall, these preliminary findings provide evidence to hypothesize an aversive, extinctionresistant experience might change the emotional load, affecting the ability to adapt walking.
\end{abstract}

Key words: Aversive Memory; Stress; Walking Adaptability; Skilled Walking. 


\section{Introduction}

The ability to adjust the feet to environmental context is a crucial aspect of walking adaptability and is an important skill to maintain the stability of the body and prevent falls $[1,2]$. Moreover, the task of adapting walking (e.g. turning, stepping to safe footfall locations etc.) is thought to be more demanding of cognitive resources, as seen in stroke survivors and older adults [2]. In addition, previous studies in humans have also suggested the activation of primary sensorimotor cortex, prefrontal area and hippocampus are associated with an adequate walking adaptability $[3,4]$. In the context of the cognitive demands of altering walking in response to the environment [1-4], the stress/emotional state of an individual may modulate the performance of skilled/adaptable walking to a different extent than motor acts such as reaching or walking over flat level surfaces.

Whilst the connections between stress, fear and movement have been studied in terms of freezing and fight-or-flight behaviors in different animal models, recent findings have suggested stressful/aversive experiences can also influence skilled sensorimotor functions in the short and longterm [5-8]. For example, acute and chronic stress and corticosterone infusion impair skilled movements [6,8] as well as the normal kinematic pattern during forelimb reaching in rodents [7]. Indeed, several reports support the hypothesis that aversive experiences may affect the locomotor system in both the short and long-term, although the mechanisms are not fully understood [6-10].

Consolidation and evocation of aversive memories require a complex organized neuroanatomical pathway, involving structures such as the amygdala, hippocampus [5] and, more recently discovered, the cerebellum [11], an important structure involved in the learning and performance of visually-cued movements (such as adjusting steps in response to what we see in the environment) [12]. Despite evidence of overlap of cognitive and emotional networks while adapting walking [4], little attention has been given to the effects of suffering an aversive experience, which generates an extinction-resistant memory, on the walking adaptability/skilled walking performance. Thus, the proposed study aims to assess, preliminarily, whether suffering an aversive experience that generates an extinction-resistant memory $[5,13]$ can trigger impairments in walking adaptability of adult rats. $[6,8,14]$

\section{Methods}

Male Wistar rats (3 months old, $\pm 300 \mathrm{~g}$ ) were obtained from the Centro de Modelos Biológicos Experimentais (CeMBE) of the Pontifícia Universidade Católica do Rio Grande do Sul (our regular provider). The animals were housed four to a cage and kept with free access to food and water, under a 12-h light/dark cycle (lights on at 7:00 a.m.) with room temperature maintained at $22-24{ }^{\circ} \mathrm{C}$. All 
procedures were in accordance with the National Institute of Health's Guide for the Care and Use of Laboratory Animals and with the Brazilian Council for Animal Experiments Control (Concea). The Animal Bioethics Committee of the Pontifical Catholic University of Rio Grande do Sul approved the study protocol (number 15/00442).

Inescapable footshocks (IF) are an aversive and stressful event for rodents that is widely used to model fear conditioning and aversive memory in rats $[5,10,15,16]$. IF has been shown to elicit dysregulation of the hypothalamus-pituitary-adrenal (HPA) axis and the neurotransmitter systems as well as functional and/or structural changes in the hippocampus, amygdala, prefrontal cortex, locus coeruleus, and ventral tegmental area $[5,13,16]$.

The fear-conditioning chamber (Panlab, Barcelona, Spain) is made of aluminum walls $(35 \times 35 \times 35 \mathrm{~cm})$ and a clear front access panel. The floor of the chamber consists of parallel grid bars made from stainless-steel, spaced $0.8 \mathrm{~mm}$ apart. The grid is connected to a device that delivers the footshocks and the apparatus was placed inside a sound-attenuating box (Panlab, Barcelona, Spain) with a ventilating fan. Rats were placed, one at a time, into the fear-conditioning chamber, and left to explore it for two minutes, then two intense electrical footshocks $(1 \mathrm{~mA}, 60 \mathrm{~Hz}, 20 \mathrm{sec})$ were delivered at a $30 \mathrm{sec}$ interval. Thirty seconds after the last footshock, the animal was removed from the conditioning chamber and returned to its home cage. The animals in the sham group (control) underwent the described procedure, but did not receive the footshocks. The chamber was cleaned with $70 \%$ ethanol before and after each use. This protocol was adapted from previous studies [5, 13, 17].

In the first part of the experiment, to assess extinction-resistance of the aversive memory [5, $13,17]$, we randomly assigned rats into two groups: shock $(N=7)$ and sham $(N=9)$. Twenty-four (S1) and 48 hours (S2) after the exposure to IF, the rats were placed back in the same apparatus for ten minutes of extinction training (no footshocks were given). The following day (72 hours after the traumatic protocol), the animals were placed back in the apparatus for a three minute retention test (also without footshocks) (Figure 1). The time (sec) of freezing behavior (no visible movement except for respiration) was assessed during the first and last three minutes of the extinction training, and throughout the three minutes the retention test. Freezing behavior was scored by two independent researchers and converted into a time percentage $[13,17]$.

After confirming IF induced an extinction-resistant memory (first part of the experiment), we evaluated its effects on walking adaptability using the ladder rung walking test $[8,14]$. This test provides an evaluation of skilled walking by measuring forelimb and hindlimb placement to ladder 
rungs (which demand accurate foot placement); stepping, and inter-limb co-ordination, reflecting skilled walking performance, and providing an indicator of walking adaptability in rats [14]. The ladder rung walking apparatus consists of a $1 \mathrm{~m}$ long horizontal ladder placed $30 \mathrm{~cm}$ above the table with stainless-steel rungs that can be repositioned $(3.0 \mathrm{~mm}$ diameter $1-3 \mathrm{~cm}$ apart); the arrangement varied between the trials to prevent the animals learning the rung positions and reinforce the need of walking adaptation, as previously described [14].

In the second part of the experiment, the animals from the shock $(N=12)$ and sham $(N=8)$ groups were tested in the ladder rung walking test at baseline (before the footshocks) and post-shock (72 hours after the shocks). We assumed the time-window between delivering the footshocks and the walking adaptability assessment ( $72 \mathrm{~h}$ post-shock) was large enough to reduce the acute effects of stress hormones, i.e., corticosterone, as suggested by previous studies [18, 19]. Thus, walking adaptability would have been assessed while classical stress-related hormones were at normal levels, especially because the animals were familiarized with the ladder waking apparatus before the footshocks, preventing a novelty-related stress [20]. Two days prior to baseline evaluation, the rats were trained (four trials a day) to cross the apparatus, as an acclimation procedure [14]. During the testing sessions, the rats were filmed crossing the apparatus. We assessed the following endpoints: a) skilled walking performance score (SWPS) (\% baseline performance); b) number of valid gait cycles; c) immobility time (the time spent immobile during the trial); d) number of stops during the trial; e) trial time (time spent to cross the apparatus); and f) walking speed during the trial $(\mathrm{cm} / \mathrm{sec})$.

Additionally, we performed the elevated plus maze test $(\approx 3$ hours prior to the post-shock ladder rung walking test) to evaluate the animals' anxiety-like behavior [15]. After the last ladder rung walking test evaluation, animals received a lethal injection of sodic pentobarbital associated with lidocaine $(10 \mathrm{mg} / \mathrm{mL})$ (i.p.) [16].

\section{Statistical Analysis}

The statistical analyses were performed using SPSS 17.0 (Statistical Package for the Social Sciences, USA). One-way ANOVA or repeated measures ANOVA were used to detect group differences, as appropriate. When nonparametric, logarithm transformation was applied before performing the tests. Pearson's or Spearman's correlation coefficient tests were used to correlate endpoints. Data are expressed according to their statistical distribution: parametric as mean \pm standard error and nonparametric as median, 2.5-97.5 percentile and range (minimum and maximum). The results were considered significant when $P \leq 0.05$. 


\section{Results}

The results of experiment, part 1 are shown in the Figure 2. We observed the main effects of "group" $\left(\mathrm{F}_{1,15}=465.83 / P=0.0001\right)$ and "time and group" interaction $\left(\mathrm{F}_{4,15}=4.55 / P=0.003\right)$ for freezing behavior. No "time" effect was observed $\left(\mathrm{F}_{4,15}=0.76 / P=0.55\right)$. In the shock group, the percentage of time engaged in freezing increased when compared to the sham group at S1 and S2 and at the retention test $(P<0.001)$. Furthermore, for the shock group, the percentage of time engaged in freezing remained unchanged over time (S1, S2 and retention test).

On the ladder rung walking test outcomes (Figure 3A-F) we observed the shock group performed poorly, on the skilled walking performance score, when compared to the sham group three days after the footshock protocol $\left(\mathrm{F}_{1,19}=30.03\right.$ / $\left.P=0.0001\right)$. Moreover, shock group tended to reduce the number of valid gait cycles after the footshock protocol (time: $\mathrm{F}_{1,19}=0.03$ / $P=0.85$; group: $\mathrm{F}_{1,19}=3.94 / P=0.06$; time*group: $\mathrm{F}_{1,19}=23.93 / P=0.0001$ ). No relevant changes were observed for “immobility time" (time: $\mathrm{F}_{1,19}=0.27 / P=0.61$; group: $\mathrm{F}_{1,19}=1.61 / P=0.22$; time*group: $\mathrm{F}_{1,19}=0.58$ / $P=0.81$ ); "number of stops during the trial" (time: $F_{1,19}=0.16 / P=0.90$; group: $F_{1,19}=1.33 / P=0.26$; time*group: $\mathrm{F}_{1,19}=0.11 / P=0.91$ ); "trial time" (time: $\mathrm{F}_{1,19}=0.18 / P=0.67$; group: $\mathrm{F}_{1,19}=1.63 / P=0.21$; time*group: $\mathrm{F}_{1,19}=0.63 / P=0.80$ ); and "walking speed" (time: $\mathrm{F}_{1,19}=0.66$ / $P=0.42$; group: $\mathrm{F}_{1,19}=0.92$ / $P=0.35$; time*group: $\mathrm{F}_{1,19}=0.30 / P=0.58$ ). Together, these results suggest aversive, extinctionresistant memory could induce changes in walking adaptability, resulting in a worse adaptability while walking on the rungs of the apparatus.

On the elevated plus maze test, (Figure 3G-I) we found a statistical difference in the "total crossings" $\left(F_{1,19}=7.85 / P=0.01\right)$, suggesting the shock group tended to reduce the exploration of the entire apparatus in comparison with sham animals. However, no additional changes were observed during the test (closed arms entries: $F_{1,19}=0.19 / P=0.66$; and time spent in opened arms: $F_{1,19}=1.02$ / $P=0.32)$.

When correlating the skilled walking score performance and the elevated plus maze data we observed the former is positively correlated with "number of gait cycles" ( $\mathrm{r}=0.85$ / $P=0.0001)$; "trial time" ( $\mathrm{r}=-0.43$ / $P=0.05)$; and "total crossings" in the plus maze test $(\mathrm{r}=0.59$ / $P=0.006)$. This finding suggests walking adaptability performance may be correlated with the exploratory pattern adopted in the plus maze test. No additional significant correlations were found when comparing ladder rung walking score to the other ladder endpoints (Table 1). 


\section{Discussion}

The aim of this study was to determine whether an aversive, extinction-resistant memory could trigger impairments in walking adaptability using a footshock paradigm to induce the aversive memory [5, 15-17]. Our results show that skilled walking performance score (reflecting errors in foot placement in hind and forelimbs) was reduced following extinction-resistant aversive stimuli. Notwithstanding, this preliminary study expands the knowledge about the influence of emotional load on walking adaptability, showing the relation between an aversive memory and adapted walking. While our results demonstrate that an aversive experience influenced the skilled walking score, all the other stress-related endpoints in the ladder rung walking test (immobility time and trial time) were unaffected. The lack of between-group differences in these endpoints, particularly the immobility time, could be interpreted as evidence that reduced walking adaptability in the shock group was not associated to the classical generalization of fear, which may occur after some aversive experiences [21]. This reinforces our hypothesis that suffering an extinction-resistance aversive memory might be the basis to change the emotional load and the ability to adapt walking, consequently.

Regarding the plus maze test, we observed fewer crossings in the shock group. Previous exposure to a single session of footshocks induces long-lasting inhibition of activity in unknown environments that markedly differ from the shock context [15], which may explain the reduction in exploratory activity. Thus, the elevated plus maze data also corroborate our hypothesis the emotional load, not necessarily typical anxiety-like status, might influence locomotor control. However, given the lack of a significant relationship between skilled walking score and closed arms entries/permanence in the elevated plus maze test, the influence of a typical anxiety response on walking is less likely. Nevertheless, further research to clarify the extent to which fear/anxiety has an effect on general walking behavior as opposed to skilled/adaptable walking may specifically help determine which neural networks and control processes are required for these different locomotor patterns.

To best of our knowledge, this is the first study showing an extinction-resistant memory might trigger changes in walking adaptability, which is in agreement with the idea that movement can be affected by the emotional context $[1,6,22]$. For example, temporal features of motion kinematics are tuned by the emotional valence of the stimulus, reinforcing the premise that emotion modulates motor planning [2]. Moreover, higher self-perceived levels of stress induce changes in gait stride velocity [1] and daily stress application can impair skilled walking performance at acute, subacute and/or chronic phases through hormonal influences [6-8]. In rats, this impairment includes an increased base of support, foot rotation and reduced stride length [8]. Furthermore, cognitive demands could also 
affect walking adaptability [2], which is in agreement with our preliminary findings. Hence, whether life experiences might recruit/modulate specific walking adaptability networks according to the emotional load is matter for further research.

Whilst it is not possible to suggest the involvement of specific neural networks linking aversive memories and walking adaptability in this preliminary study, hypothetical speculations might be interesting to guide the development of further research. There is considerable evidence showing that aversive memory consolidation processes in the cerebellar vermis are long lasting and differ from those in the amygdala $[11,23]$. For instance, administration of tetrodotoxin a few days, but no later than two weeks after a fear acquisition session, disrupts memory consolidation [24]. Furthermore, fear memory consolidation encompasses cerebellar changes such as the long-term increase in synaptic efficacy between parallel fibers and Purkinje cells, while primary deficiency of these parallel fibers to the Purkinje cell synapse induces short and long-term fear memory impairment $[11,24,25]$. Thus, the cerebellum, like the hippocampus, is involved in the late phase of memory consolidation [11, 23, 24] and in walking adaptability [3]. Nonetheless, in a speculative-only point of view, we hypothesize that neural networks related to aversive memory processing might influence the sensorimotor control of walking adaptability [11, 3, 23-25]. Likewise, it is still unclear if cerebellar traumatic memory consolidation or resistance to extinction are the main triggers for sensorimotor adaptation impairments, which is also a matter for further investigation.

In addition, these preliminary results open many research perspectives. For example, it is widely known that the peak of neuroplasticity and basic motor skill acquisition occur in earlier stages of sensorimotor development. However, conditions common in adulthood such as ageing [3], stress [6-9] and stroke [2] can impair the ability to adapt walking. Thus, to compare the effects of suffering aversive experiences on walking adaptability in different periods, i.e. neonate, adolescent and adult, might help clarify how walking adaptability is regulated over a lifetime. Moreover, complementary experiments using different biochemical approaches could be an important next step to clarify the hypothetical connection between aversive memory and walking adaptability. For instance, modulating aversive memory formation, consolidation and reconsolidation using different drugs, i.e. protein synthesis inhibitors delivered in the amygdala and/or prefrontal cortex $[5,17]$ may provide definitive evidence to support the connection between aversive memory and walking adaptability. Furthermore, assessing dopaminergic neurotransmission and brain-derived neurotrophic factor pathways in these structures [5] might shed additional light on the regulation of the above-mentioned connection. 
One limitation of this study is that although we believe the current findings encourage further studies modulating neurochemical mechanisms that may link both issues, we cannot establish a direct cause and effect relationship between suffering an aversive, extinction-resistant memory and walking adaptability at this stage. For instance, vulnerability and protective factors for stress exposure might explain, at least in part, the present results. Thus, care should be taken in their interpretation.

Finally, this preliminary short-report provides evidence to suggest that suffering aversive experiences might change the emotional load, affecting the ability to adapt walking. However, further studies evaluating which neural networks and/or neurochemical factors are involved in walking adaptability are needed to clarify whether the emotional-load is a significant factor affecting this behavior.

\section{Conflict of interest}

The authors declare that there are no conflicts of interest.

\section{Acknowledgments}

This research was supported by the Brazilian funding agencies: Conselho Nacional de Pesquisa e Desenvolvimento (CNPq), Coordenação de Aperfeiçoamento de Pessoal de Nível Superior (CAPES) and Fundação de Apoio à Pesquisa do Estado do Rio Grande do Sul (FAPERGS). 


\section{References}

[1] P.O. Esteves, L.A. Oliveira, A.A. Nogueira-Campos, G. Saunier, T. Pozzo, J.M. Oliveira, E.C. Rodrigues, E. Volchan, C.D. Vargas, Motor planning of goal-directed action is tuned by the emotional valence of the stimulus: a kinematic study, Sci. Rep. 6 (2016) 28780. doi: 10.1038/srep28780

[2] K.L. Hollands, D. Agnihotri, S.F. Tyson, Effects of dual task on turning ability in stroke survivors and older adults, Gait Post. 40(4) (2014) 564-9. doi: 10.1016/j.gaitpost.2014.06.019

[3] H. Shimada, K. Ishii, K. Ishiwata, K. Oda, M. Suzukawa, H. Makizako, T. Doi, T. Suzuki, Gait adaptability and brain activity during unaccustomed treadmill walking in healthy elderly females, Gait. Post. 38(2) (2013) 203-8. doi: 10.1016/j.gaitpost.2012.11.008

[4] K.L. Koenraadt, E.G. Roelofsen, J. Duysens, N.L. Keijsers. Cortical control of normal gait and precision stepping: an fNIRS study. Neuroimage 15(85) (2014) 415-22. doi: 10.1016/j.gaitpost.2012.11.008.

[5] I. Izquierdo, C.R. Furini, J.C. Myskiw, Fear Memory, Physiol. Rev. 96(2) (2016) 695-750. doi: 10.1152/physrev.00018.2015

[6] G.A. Metz, N.M. Jadavji, L.K. Smith, Modulation of motor function by stress: a novel concept of the effects of stress and corticosterone on behavior, Eur. J. Neurosci. 22(5) (2005) 1190-200. doi: 10.1111/j.1460-9568.2005.04285.x [7] N.M. Jadavji, R.D. Supina, G.A. Metz, Blockade of mineralocorticoid and glucocorticoid receptors reverses stressinduced motor impairments, Neuroendocrinology. 94(4) (2011) 278-90. doi: 10.1159/000329988

[8] G.A. Metz, M.E. Schwab, H. Welzl, The effects of acute and chronic stress on motor and sensory performance in male Lewis rats, Physiol. Behav. 72(1-2) (2001) 29-35. doi: 10.1016/S0031-9384(00)00371-1

[9] R. Holtzer, C. Schoen, E. Demetriou, J.R. Mahoney, M. Izzetoglu, C. Wang, J. Verghese, Stress and gender effects on prefrontal cortex oxygenation levels assessed during single and dual-task walking conditions, Eur. J. Neurosci. 45(5) (2016) 660-670. doi: 10.1111/ejn.13518

[10] A. Der-Avakian, R.R. Rozeske, S.T. Bland, L.R. Watkins, S.F. Maier, The effects of a single session of inescapable tailshock on the subsequent locomotor response to brief footshock and cocaine administration in rats,

Psychopharmacol. (Berl) 191(4) (2007) 899-907. doi: 10.1007/s00213-006-0677-8

[11] B. Sacchetti, B. Scelfo, P. Strata, The cerebellum: synaptic changes and fear conditioning, Neuroscientist. 11(3) (2005) 217-27. doi: 10.1177/1073858405276428

[12] C.I. De Zeeuw, M.M. Ten Brinke, Motor Learning and the Cerebellum, Cold. Spring. Harb. Perspect. Biol. 7(9) (2015) a021683. doi: 10.1101/cshperspect.a021683

[13] A. Izquierdo, C.L. Wellman, A. Holmes, Brief uncontrollable stress causes dendritic retraction in infralimbic cortex and resistance to fear extinction in mice, J. Neurosci. 26(21) (2006) 5733-8. doi: 10.1523/JNEUROSCI.0474-06.2006 [14] G.A. Metz, I.Q. Whishaw, Cortical and subcortical lesions impair skilled walking in the ladder rung walking test: a new task to evaluate fore- and hindlimb stepping, placing, and co-ordination, J. Neurosci. Methods. 115(2) (2002) 16979. doi: 10.1016/S0165-0270(02)00012-2

[15] N. Daviu, S. Fuentes, R. Nadal, A. Armario, A single footshock causes long-lasting hypoactivity in unknown environments that is dependent on the development of contextual fear conditioning, Neurobiol. Learn. Mem. 94(2) (2010) 183-90. doi: 10.1016/j.nlm.2010.05.005

[16] L. Saur, P.P. Baptista, P.B. Bagatini, L.T. Neves, R.M. de Oliveira, S.P. Vaz, K. Ferreira, S.A. Machado, R.G. Mestriner, L.L. Xavier, Experimental Post-traumatic Stress Disorder Decreases Astrocyte Density and Changes Astrocytic Polarity in the CA1 Hippocampus of Male Rats, Neurochem. Res. 41(4) (2016) 892-904. doi: 10.1007/s11064-015-1770-3

[17] J. de Carvalho Myskiw, C.R. Furini, B. Schmidt, F. Ferreira, I. Izquierdo, Extinction learning, which consists of the inhibition of retrieval, can be learned without retrieval, Proc. Natl. Acad. Sci. USA. 112(2) (2015) E230-3. doi: 10.1073/pnas.1423465112

[18] M.I. Cordero, C. Venero, N.D. Kruyt, C. Sandi, Prior exposure to a single stress session facilitates subsequent contextual fear conditioning in rats. Evidence for a role of corticosterone, Horm. Behav. 44(4) (2003) 338-45. doi: 10.1016/S0018-506X(03)00160-0

[19] V.P. Bakshi, K.M. Alsene, P.H. Roseboom, E.E. Connors, Enduring sensorimotor gating abnormalities following predator exposure or corticotropin-releasing factor in rats: a model for PTSD-like information-processing deficits?, Neuropharmacol. 62(2) (2012) 737-48. doi: 10.1016/j.neuropharm.2011.01.040

[20] C. Sandi, C. Venero, C. Guaza, Novelty-related rapid locomotor effects of corticosterone in rats, Eur. J. Neurosci. 8(4) (1996) 794-800. doi: 10.1111/j.1460-9568.1996.tb01264.x

[21] J.E. Dunsmoor, M.C. Kroes, S.H. Braren, E.A. Phelps, Threat intensity widens fear generalization gradients, Behav. Neurosci. 131(2) (2017) 168-175. doi: 10.1037/bne0000186

[22] S. Aybek, T.R. Nicholson, O. O'Daly, F. Zelaya, R.A. Kanaan, A.S. David, Emotion-motion interactions in conversion disorder: an FMRI study, PLoS One 10(4) (2015) e0123273. doi: 10.1371/journal.pone.0123273 
[23] M. Mintz, Y. Wang-Ninio, Two-stage theory of conditioning: involvement of the cerebellum and the amygdala, Brain. Res. 897(1-2) (2001) 150-6. doi: 10.1016/S0006-8993(01)02111-4

[24] B. Sacchetti, E. Baldi, C.A. Lorenzini, C. Bucherelli, Cerebellar role in fear-conditioning consolidation, Proc. Natl. Acad. Sci. USA. 99(12) (2002) 8406-11. doi: 10.1073/pnas.112660399

[25] D. Heck, F. Sultan, Cerebellar structure and function: making sense of parallel fibers, Hum. Mov. Sci. 21(3) (2002)

411-21. doi: 10.1016/S0167-9457(02)00123-9

\section{Figure Legends}

Figure 1. Experimental design of experiments 1 and 2. S1: first session of extinction training; S2: second session of extinction training; RT: retention test.

Figure 2. Freezing $(\%)$ in the extinction training and memory retention test. $* * *$ Difference between sham and shock groups at $P<0.0001$. S1: first session of extinction training, S2: second session of extinction training, RT: retention test.

Figure 3. A-F: Ladder rung walking test outcomes (baseline and post-shock). ***Difference betweeen sham and shock groups at $P<0.0001$; G-I: Plus Maze test performance (post-shock). *Difference betweeen sham and footschok groups at $P<0.05$. 